\title{
The Impact of Pedigree Structure on Heritability Estimates
}

\author{
Claus Thorn Ekstrøm
}

Statistics, Department Of Natural Sciences, Faculty of LIFE Sciences, University of Copenhagen, Frederiksberg, Denmark

\section{Key Words}

Heritability · Precision - Variance components - Pedigree structure

\begin{abstract}
Heritability measures the familial aggregation of a disease or trait and a non-zero heritability suggests that a genetic component may be present. Reliable heritability estimates are necessary in the planning phase of a linkage or genetic association study but often these estimates are obtained from other studies where the composition of pedigrees may be different from the study that is prepared. The impact of pedigree structure on precision and accuracy of heritability estimates is examined for data and models both with and without dominance effects. Analytical and simulation results find that for purely additive genetic effects all but the simplest pedigree structures provide the same information about the heritability of a quantitative trait. In the presence of dominance effects there is a substantial difference in the precision obtained by different pedigree structures.
\end{abstract}

Copyright $\odot 2009$ S. Karger AG, Basel

\section{Introduction}

The concept of heritability plays an important role in genetic epidemiology since familial aggregation of a disease or trait is a necessary condition to infer a genetic component. Heritability estimation is usually considered the first step in unravelling the genetic basis of a disease or trait and countless studies present heritability estimates for different traits or diseases. Subsequent steps in gene identification often rely heavily on heritability estimates from pilot studies or from published studies since these estimates help determine the required sample size in order for the study to have a certain power to identify the susceptibility gene.

Reliable heritability estimates are important in the planning phase of a linkage study or genetic association study since these studies are irrelevant unless a substantial genetic variation is present in the population. However, it has only rarely been examined how different pedigree structures influence the heritability estimates and if estimates from a particular set of pedigree structures are directly applicable to a study employing a different set of pedigree structures.

In a recent paper Hsu et al. [2005] find different heritability estimates for pulse pressure in three different populations and conclude - after a resampling approach to match pedigree sizes for the three populations - that pedigree structure is one of the important factors affecting heritability estimates. Their results contradict earlier results by Mallinckrodt et al. [1997] who found hardly any difference between the confidence intervals of the heritability when estimates were obtained from simulated data of random pedigree structures or fixed pedigree structures.

\section{KARGER \\ Fax +4161306 1234 E-Mail karger@karger.ch} www.karger.com (c) 2009 S. Karger AG, Basel

0001-5652/09/0684-0243\$26.00/0

Accessible online at:

www.karger.com/hhe
Claus Thorn Ekstrøm

Statistics, Department of Natural Sciences

Faculty of LIFE Sciences, University of Copenhagen

Thorvaldsensvej 40, DK-1871 Frederiksberg C (Denmark)

Tel. +45 35332 341, Fax +45 35282 350, E-Mail ekstrom@life.ku.dk 
The purpose of the present paper is twofold: First, we wish to examine the impact of pedigree structure on the precision and accuracy of heritability estimates and secondly we wish to determine the necessary sample size to obtain a given precision of the heritability estimate. In the next section we discuss the multivariate Gaussian variance component model for heritability estimation of quantitative traits and how the heritability precision is influenced by the variance of the variance components. We illustrate the impact of different pedigree structures on the heritability estimates through a set of simulations before we discuss the design requirements for obtaining reliable heritability results from a pilot study.

\section{Methods}

Let $y_{i}$ be the vector of observed phenotypes for pedigree $i$ comprising $n_{i}$ individuals. The classical multivariate normal additive polygenic model [Lange et al., 1976; Hopper and Mathews, 1982] assumes that the mean of the observed phenotype is given by $\mu_{i}=\mathbf{E}\left(y_{i}\right)=f_{\beta}\left(x_{i}\right)$ and depends on $l$ parameters $\left(\beta_{1}, \ldots, \beta_{l}\right)$ through a known function $f$ and a set of covariates $x_{i}$. Furthermore, the variance-covariance matrix, $\Omega$, is a linear combination of independent matrices with known covariance structure,

$$
\Omega_{i}=\sigma_{a}^{2} 2 \Phi_{i}+\sigma_{I}^{2} \mathbf{I}_{i} .
$$

$\sigma_{a}^{2}$ and $\sigma_{I}^{2}$ are the variance component effects corresponding to a residual additive effect and a non-shared environmental effect, respectively. The $(j, k)$ th element of the kinship coefficient matrix, $\Phi^{j k}$, is defined as the probability that an allele drawn at random from an arbitrary locus in individual $j$ is identical by descent to an allele drawn at random from the same locus in individual $k$. $\mathbf{I}$ is the identity matrix. The log-likelihood of pedigree $i$ becomes

$$
\log L=-\frac{n_{i}}{2} \log (2 \pi)-\frac{1}{2} \log \left(\left|\Omega_{i}\right|\right)-\frac{1}{2}\left(y_{i}-\mu_{i}\right)^{\top} \Omega_{i}^{-1}\left(y_{i}-\mu_{i}\right) .
$$

The polygenic model defined by (1) and (2) form the basis of many linkage studies of quantitative traits, and has been extended to accommodate for example dominance effects, shared environmental effects [Hopper and Mathews, 1982] and multiple genotyped loci [Almasy and Blangero, 1998].

The (narrow) sense heritability is defined as the proportion of the total phenotypic variance that is attributable to additive genetic effects [Falconer and Mackay, 1996]

$$
h^{2}=\eta\left(\sigma_{a}^{2}, \sigma_{I}^{2}\right)=\frac{\sigma_{a}^{2}}{\sigma_{a}^{2}+\sigma_{I}^{2}} .
$$

The bias of the estimator is defined as the difference between the mean heritability estimate and the true heritability value, $\mathbf{E} \hat{h}^{2}-h^{2}$

The heritability estimate has asymptotic variance given by the matrix product

$$
V\left(\hat{h}^{2}\right)=\eta^{\prime}\left(\sigma_{a}^{2}, \sigma_{I}^{2}\right) \Gamma \eta^{\prime}\left(\sigma_{a}^{2}, \sigma_{I}^{2}\right)^{\top},
$$

where $\Gamma=\operatorname{Var}\left(\hat{\sigma}_{a}^{2}, \hat{\sigma}_{I}^{2}\right)$ is the asymptotic variance-covariance matrix of the two variance components and where the partial derivatives of $\eta$ with respect to $\sigma_{a}^{2}$ and $\sigma_{I}^{2}$ are

$$
\frac{\partial \eta}{\partial \sigma_{j}^{2}}= \begin{cases}\frac{\sigma_{I}^{2}}{\left(\sigma_{a}^{2}+\sigma_{I}^{2}\right)^{2}} & \text { when } \sigma_{j}^{2}=\sigma_{a}^{2} \\ -\frac{\sigma_{a}^{2}}{\left(\sigma_{a}^{2}+\sigma_{I}^{2}\right)^{2}} & \text { when } \sigma_{j}^{2}=\sigma_{I}^{2}\end{cases}
$$

Let $\sigma^{2}=\sigma_{a}^{2}+\sigma_{I}^{2}$ denote the total phenotypic variance for an individual. The partial derivatives in (5) then correspond to $\left(1-h^{2}\right) / \sigma^{2}$ and $-h^{2} / \sigma^{2}$ respectively. Furthermore, if we write

$$
\Gamma=\left(\begin{array}{ll}
\gamma_{11} & \gamma_{12} \\
\gamma_{12} & \gamma_{22}
\end{array}\right)
$$

we can calculate the variance from formula (4) as

$$
\begin{aligned}
& V\left(\hat{h}^{2}\right)=\gamma_{11}\left(\frac{1-h^{2}}{\sigma^{2}}\right)^{2}+2 \gamma_{12}\left(\frac{1-h^{2}}{\sigma^{2}} \frac{-h^{2}}{\sigma^{2}}\right)+\gamma_{22}\left(\frac{-h^{2}}{\sigma^{2}}\right)^{2} \\
& =\frac{1}{\left(\sigma^{2}\right)^{2}}\left(\left(h^{2}\right)^{2}\left(\gamma_{11}+2 \gamma_{12}+\gamma_{22}\right)-2 h^{2}\left(\gamma_{11}+\gamma_{12}\right)+\gamma_{11}\right) .
\end{aligned}
$$

The variance of the heritability estimate is a second degree polynomial in $h^{2}$ and the quadratic coefficient must always be positive since $\left(\gamma_{11}+2 \gamma_{12}+\gamma_{22}\right)=\operatorname{Var}\left(\sigma_{a}^{2}+\sigma_{I}^{2}\right)>0$. Hence, we see that variance of the heritability is minimized when

$$
h^{2}=\frac{2\left(\gamma_{11}+\gamma_{12}\right)}{2\left(\gamma_{11}+2 \gamma_{12}+\gamma_{22}\right)}=\frac{\gamma_{11}+\gamma_{12}}{\gamma_{11}+2 \gamma_{12}+\gamma_{22}}
$$

In general, however, we do not have any control over the true population heritability and we cannot design a study that will achieve the minimum variance. Instead we need to investigate how the pedigree structure influences the precision of the variance components since they determine the sample variance through (7).

A well-known result from asymptotic theory states that the variance of the variance component maximum likelihood estimates, $\Gamma$, can be obtained from the inverse Fisher information matrix, $S^{-1}$, where $S$ in the present situation becomes

$$
S=\frac{1}{2}\left(\begin{array}{ll}
\operatorname{tr}\left(\Omega^{-1} 2 \Phi \Omega^{-1} 2 \Phi\right) & \operatorname{tr}\left(\Omega^{-1} 2 \Phi \Omega^{-1}\right) \\
\operatorname{tr}\left(\Omega^{-1} \Omega^{-1} 2 \Phi\right) & \operatorname{tr}\left(\Omega^{-1} \Omega^{-1}\right)
\end{array}\right),
$$

see Lynch and Walsh [1998, pp 788-789] for more detail. Thus, the pedigree structure enters the heritability estimate through $\Phi$ and $\Omega$ in (9). If we fix $\sigma_{a}^{2}$ and $\sigma_{I}^{2}$ (and hence we fix the heritability $h^{2}$ ), we can calculate $S$ for different pedigree structures and see how the structures affect $S$.

Figure 1 shows the different pedigree structures we examine in this paper. The pedigree structures range from simple (sib pair) to more complicated (second cousins family) and represent many of the pedigrees found in existing linkage datasets. Figure 2 shows the theoretical sample variance (7) for the different pedigree structures for varying values of $h^{2}$. The total number of observations is kept constant $(n=84)$ for each of the different pedigree structures such that the variances of the heritability estimates shown in figure 2 are directly comparable. Obviously the number of pedigrees will depend on the pedigree size such that a total of $n=84$ is achieved. 
Fig. 1. Pedigree trees of pedigrees examined in the paper. The pedigrees are (a) sib pair, (b) 2 offspring nuclear family, (c) 4 offspring nuclear family, (d) 6 offspring nuclear family, (e) CEPH pedigree, (f) double half sib family, (g) first cousins family and (h) second cousins family.

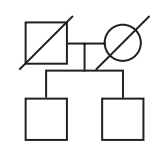

(a) Sib pair

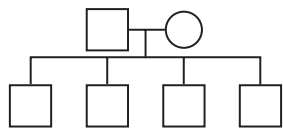

(c) Four offspring nuclear family

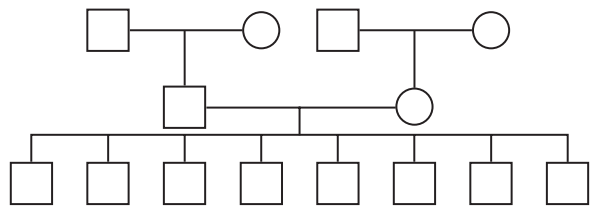

(e) $\mathrm{CEPH}$

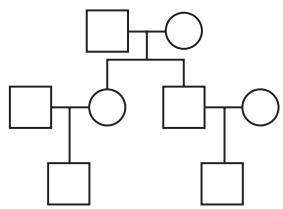

(g) First cousins

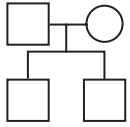

(b) Two offspring nuclear family

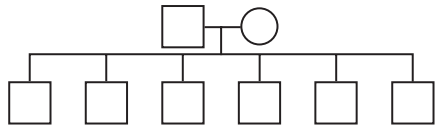

(d) Six offspring nuclear family

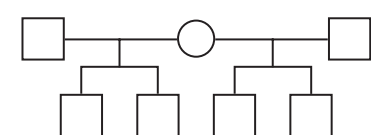

(f) Double half sib family

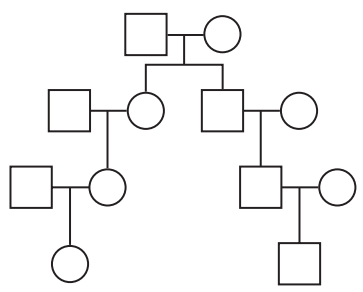

(h) Second cousins
Fig. 2. Theoretical variance of heritability estimate as a function of true heritability, $h^{2}$, for eight different pedigree structures (a)-(h) (see fig. 1). A total of 84 individuals are assumed to be in each dataset.

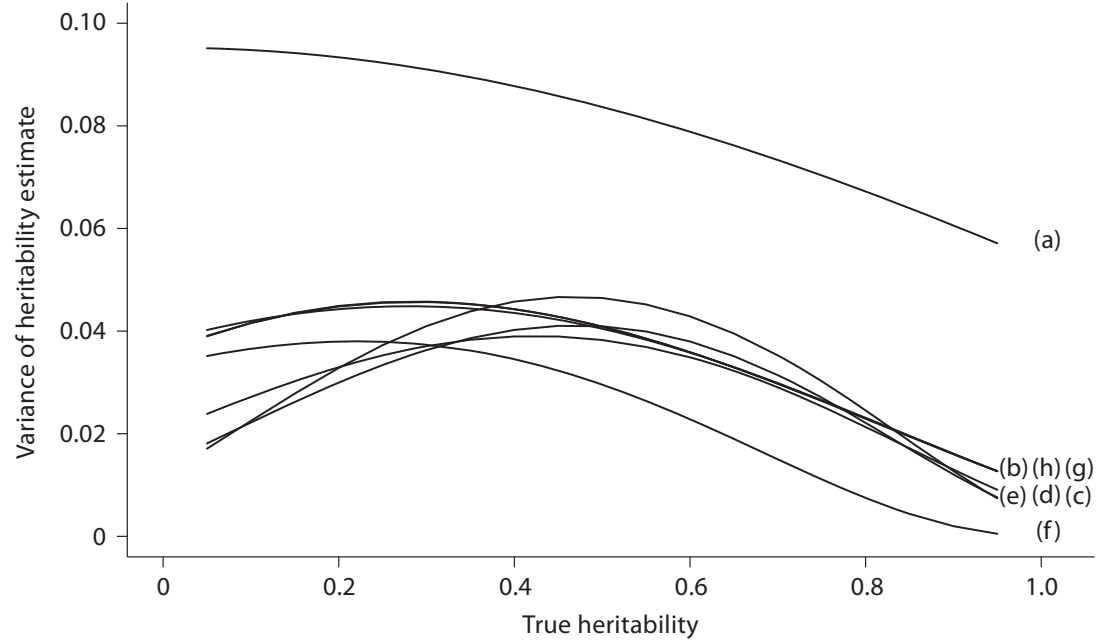


The results from figure 2 suggest that there is hardly any difference in precision of heritability estimates for different pedigree structures except for sib pairs (a) that have a relatively larger variance than the other pedigree structures.

\section{Dominance Effects}

The variance-covariance matrix, $\Omega$, changes to

$$
\Omega_{i}=\sigma_{a}^{2} 2 \Phi_{i}+\sigma_{d}^{2} \Delta_{7 i}+\sigma_{I}^{2} \mathbf{I}_{i}
$$

when dominance effects are included in the model. Here, $\sigma_{d}^{2}$ is the variance component corresponding to the dominance effect and $\Delta_{7 i}$ is Jacquard's condensed coefficient of identity and includes the probability of two individuals sharing two alleles identical by descent at a given locus [Jacquard, 1974].

The narrow sense heritability is defined as previously (3),

$$
h^{2}=\eta\left(\sigma_{a}^{2}, \sigma_{d}^{2}, \sigma_{I}^{2}\right)=\frac{\sigma_{a}^{2}}{\sigma_{a}^{2}+\sigma_{d}^{2}+\sigma_{I}^{2}},
$$

but when non-additive effects are present we can also consider the broad sense heritability

$$
H^{2}=\tilde{\eta}\left(\sigma_{a}^{2}, \sigma_{d}^{2}, \sigma_{I}^{2}\right)=\frac{\sigma_{a}^{2}+\sigma_{d}^{2}}{\sigma_{a}^{2}+\sigma_{d}^{2}+\sigma_{I}^{2}} .
$$

Following the same steps as before, we find the partial derivatives of (11) as

$$
\frac{\partial \eta}{\partial \sigma_{j}^{2}}=\left\{\begin{array}{ll}
\frac{\sigma_{I}^{2}}{\left(\sigma_{a}^{2}+\sigma_{d}^{2}+\sigma_{I}^{2}\right)^{2}} & \text { for } \sigma_{j}^{2} \in\left\{\sigma_{a}^{2}, \sigma_{d}^{2}\right\} \\
-\frac{\sigma_{a}^{2}}{\left(\sigma_{a}^{2}+\sigma_{d}^{2}+\sigma_{I}^{2}\right)^{2}} & \text { when } \sigma_{j}^{2}=\sigma_{I}^{2}
\end{array},\right.
$$

while the partial derivatives of the broad heritability $\left(11^{*}\right)$ are

$$
\frac{\partial \tilde{\eta}}{\partial \sigma_{j}^{2}}=\left\{\begin{array}{ll}
\frac{\sigma_{I}^{2}}{\left(\sigma_{a}^{2}+\sigma_{d}^{2}+\sigma_{I}^{2}\right)^{2}} & \text { for } \sigma_{j}^{2} \in\left\{\sigma_{a}^{2}, \sigma_{d}^{2}\right\} \\
-\frac{\sigma_{a}^{2}+\sigma_{d}^{2}}{\left(\sigma_{a}^{2}+\sigma_{d}^{2}+\sigma_{I}^{2}\right)^{2}} & \text { when } \sigma_{j}^{2}=\sigma_{I}^{2}
\end{array} .\right.
$$

We can then proceed exactly as before and derive the formula for the variance of the heritability, which for the narrow sense heritability (11) becomes

$$
\begin{aligned}
V\left(\hat{h}^{2}\right)= & \gamma_{11}\left(\frac{1-h^{2}}{\sigma^{2}}\right)^{2}-\frac{h^{2}\left(1-h^{2}\right)}{\left(\sigma^{2}\right)^{2}}\left(\gamma_{21}+\gamma_{12}+\gamma_{31}+\gamma_{13}\right) \\
& +\left(\frac{h^{2}}{\sigma^{2}}\right)^{2}\left(\gamma_{22}+\gamma_{32}+\gamma_{23}+\gamma_{33}\right)
\end{aligned}
$$

and for the broad sense heritability becomes

$$
\begin{aligned}
V\left(\hat{H}^{2}\right)= & \left(\gamma_{11}+\gamma_{21}+\gamma_{12}+\gamma_{22}\right)\left(\frac{1-h^{2}}{\sigma^{2}}\right)^{2} \\
& -\frac{h^{2}\left(1-h^{2}\right)}{\left(\sigma^{2}\right)^{2}}\left(\gamma_{31}+\gamma_{32}+\gamma_{13}+\gamma_{23}\right)+\left(\frac{h^{2}}{\sigma^{2}}\right)^{2} \gamma_{33} .
\end{aligned}
$$

In both (13) and $\left(13^{*}\right)$ we have extended the variance-covariance matrix (6) to $\mathbb{R}_{3}^{3}$, and we can insert the corresponding information from the inverse Fisher matrix. The results are shown in figure 3 where the variance of the narrow and broad sense heritabilities are compared for different dominance effects. Clearly, the variance of the narrow sense heritability is much smaller than the broad sense heritability, but figure 3 also shows that while there is hardly any difference in variance of narrow sense heritability for the different pedigrees (top panels in fig. 3), there are quite noticeable differences in broad sense heritability (bottom panels). Large pedigrees containing little information about dominance deviance (e.g., pedigrees (g) and (h) from fig. 1) have a markedly larger variance than smaller pedigrees. It is also worth noting that the variance generally increases when the proportion of the heritability that is due to dominance effects increases.

\section{Simulation Study}

We use a set of simulations to investigate the simultaneous impact of pedigree structure and sample size on accuracy (bias) and precision of heritability estimates. For each pedigree structure we simulated phenotypic data using the multivariate Gaussian polygenic model (2), where $\sigma_{a}^{2}$ and $\sigma_{I}^{2}$ were fixed such that $\sigma_{a}^{2}+\sigma_{I}^{2}=1$ and where $\sigma_{a}^{2}$ varied to obtain true heritabilities of $0.1,0.33$ and 0.5. To investigate the influence of sample size we simulated datasets with either $60,120,240,360,600$ or 1200 individuals. This setup may result in different number of pedigrees for the eight pedigree structures examined but it keeps the sample size constant. One thousand datasets were simulated for each combination of pedigree structure, sample size and heritability.

Figure 4 shows the mean estimated heritability and the root mean squared error for the three different heritability levels and the six different sample sizes. The upper left panel shows the mean estimated heritabilities for all three heritability levels, and - not surprisingly - the bias decreases noticeably with increasing sample size. The other three panels show the root of the mean squared error (MSE) for different true heritability values. The MSE increases with heritability but decreases with sample size.

The estimated mean squared error is practically identical for all combinations of pedigree type and heritability except for the sib pair pedigree structure (a) that are shown with bold lines on all four panels in figure 4 . The sib pairs show a large bias for small sample sizes and a low heritability of 0.1 and have markedly higher MSE. The larger MSE could also be seen on figure 2, where the variance curve for the sib pair pedigrees are substantially higher than the other variance curves.

\section{Dominance Effects}

We investigate the impact of dominance effects in two situations: First we generate data from the dominance 


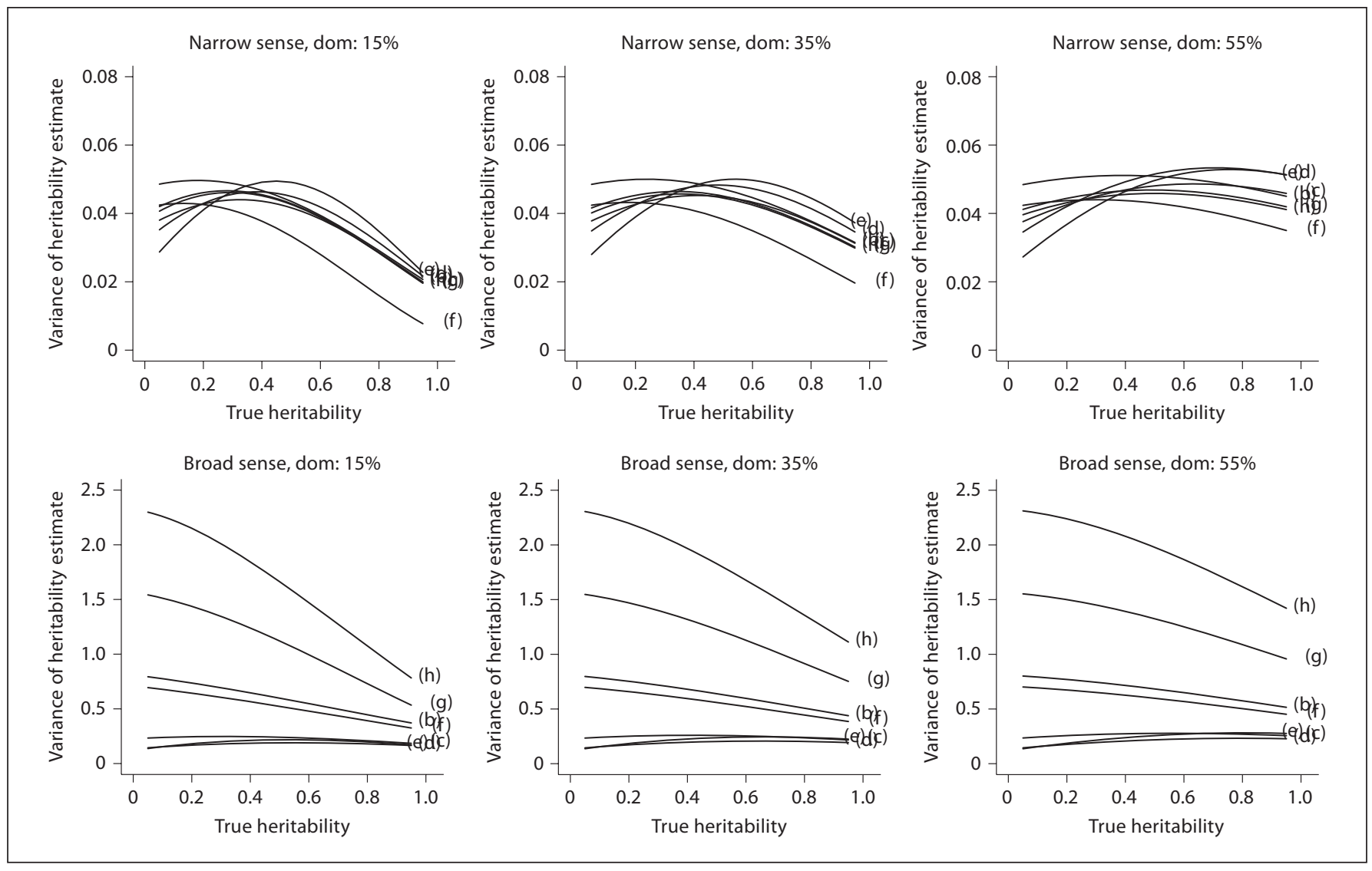

Fig. 3. Theoretical variance of heritability estimate as a function of true heritability, $h^{2}$, for seven different pedigree structures (b)-(h) (see fig. 1). A total of 84 individuals are assumed to be in each dataset. Top panels show the narrow sense heritability while bottom panels are broad sense heritability. From left to right the dominance deviance effect constitutes 15,35 and $55 \%$ of the total heritability, respectively.

Fig. 4. Mean heritability estimates and root mean squared error of the heritability estimates for eight different pedigree structures ((a)-(h) in fig. 1) and three levels of heritability $\left(h^{2}=0.1,0.33\right.$ or 0.5$)$. The upper left picture shows the mean heritability for all three heritability levels. The bold lines correspond to the sib pair pedigree structure (a). All results are based on 1000 simulated datasets.

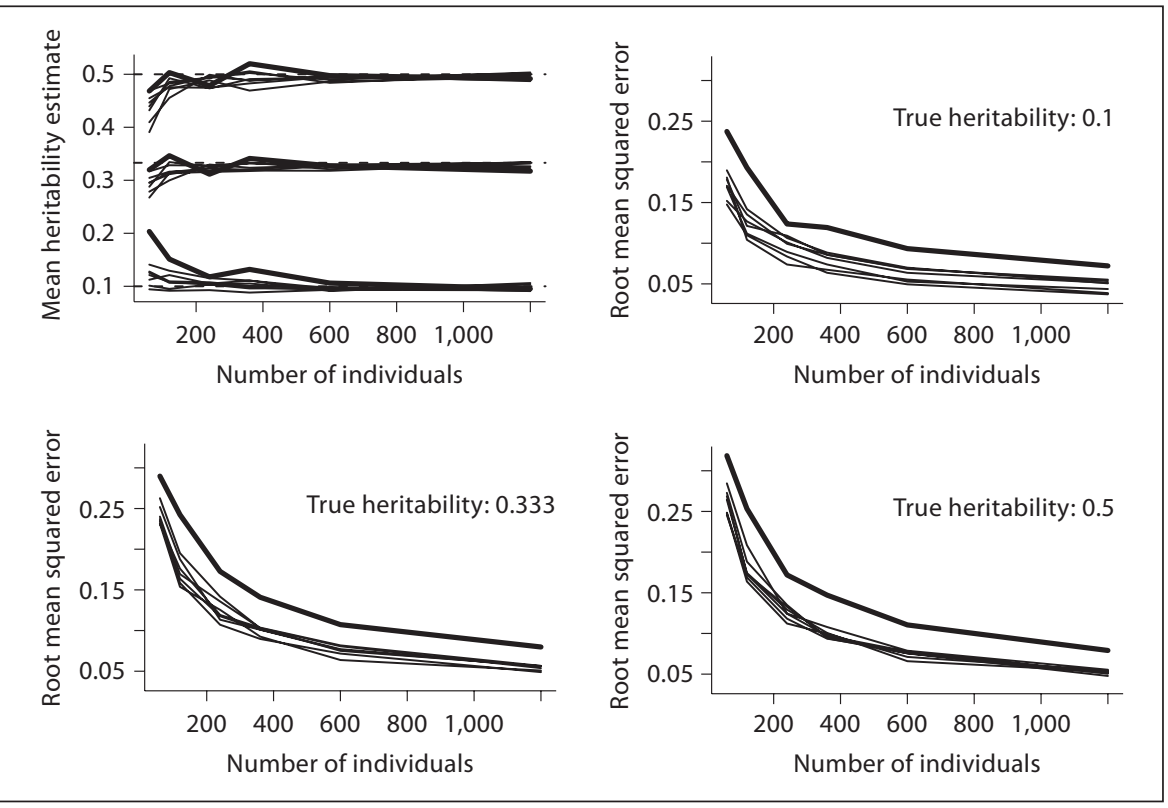




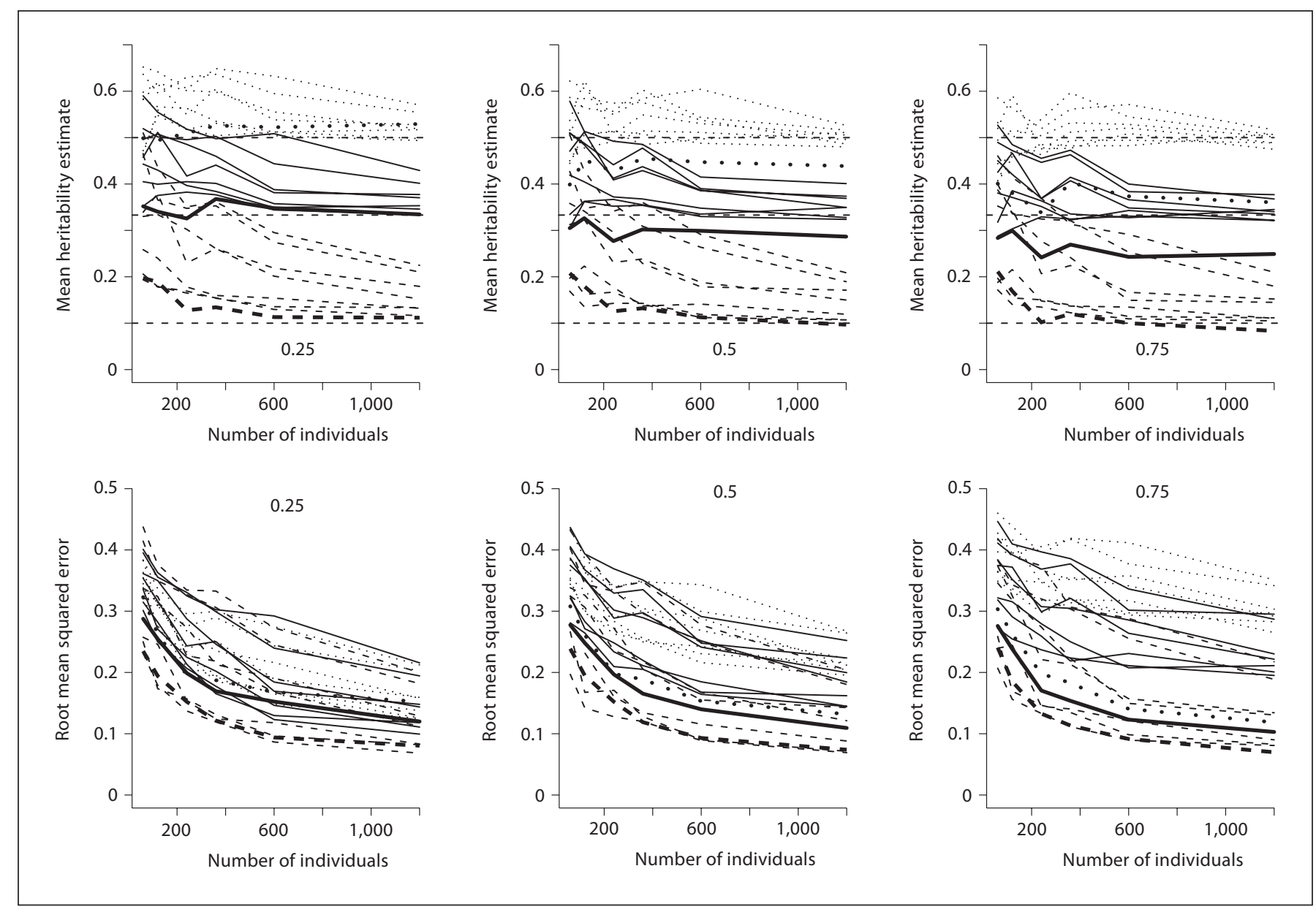

Fig. 5. Mean heritability estimates and root mean squared error of the broad sense heritability estimates for eight different pedigree structures (a)-(h) (see fig. 1) and three levels of heritability $\left(h^{2}=0.1\right.$ (solid line), 0.33 (dashed line) and 0.5 (dotted line)). Simulated data include a dominance effect accounting for 25, 50 or 75\% of the total genetic variation (columns 1-3 respectively). The bold lines correspond to the sib pair pedigree structure (a). All results are based on 1000 simulated datasets.

model (10) and also use the correct model to estimate the variance components. This enables us to evaluate the bias in heritability estimates and compare the MSE for different pedigree types. Secondly, we generate data from the dominance model (10) but use the (incorrect) additive model (1) to estimate the parameters to examine how a misspecification of the model will influence the estimates, when a dominance effect is not modeled.

Figure 5 shows the mean heritability estimates and root mean squared error of the broad sense heritability for data generated and estimated using the dominance model (10). There is hardly any difference in root mean squared error for the different pedigrees structures but figure 5 suggests that sample size has a substantial influence on heritability bias for small sample sizes. The figure also shows that a large number of pedigrees may be needed to achieve a reasonable accuracy in the heritability estimates. The two pedigree structures that perform consistently worst are the first and second cousins pedigrees (pedigrees (g) and (h) in fig. 1).

Figure 6 shows the result when data are generated from a model with a dominant genetic component but where an additive model is used to estimate the (narrow sense) heritability. In this case there is virtually no impact of pedigree structure on root mean squared error or heritability estimates but there is a considerable bias and all pedigrees underestimate the true heritability. The downward bias increases with increasing dominant genetic component. 


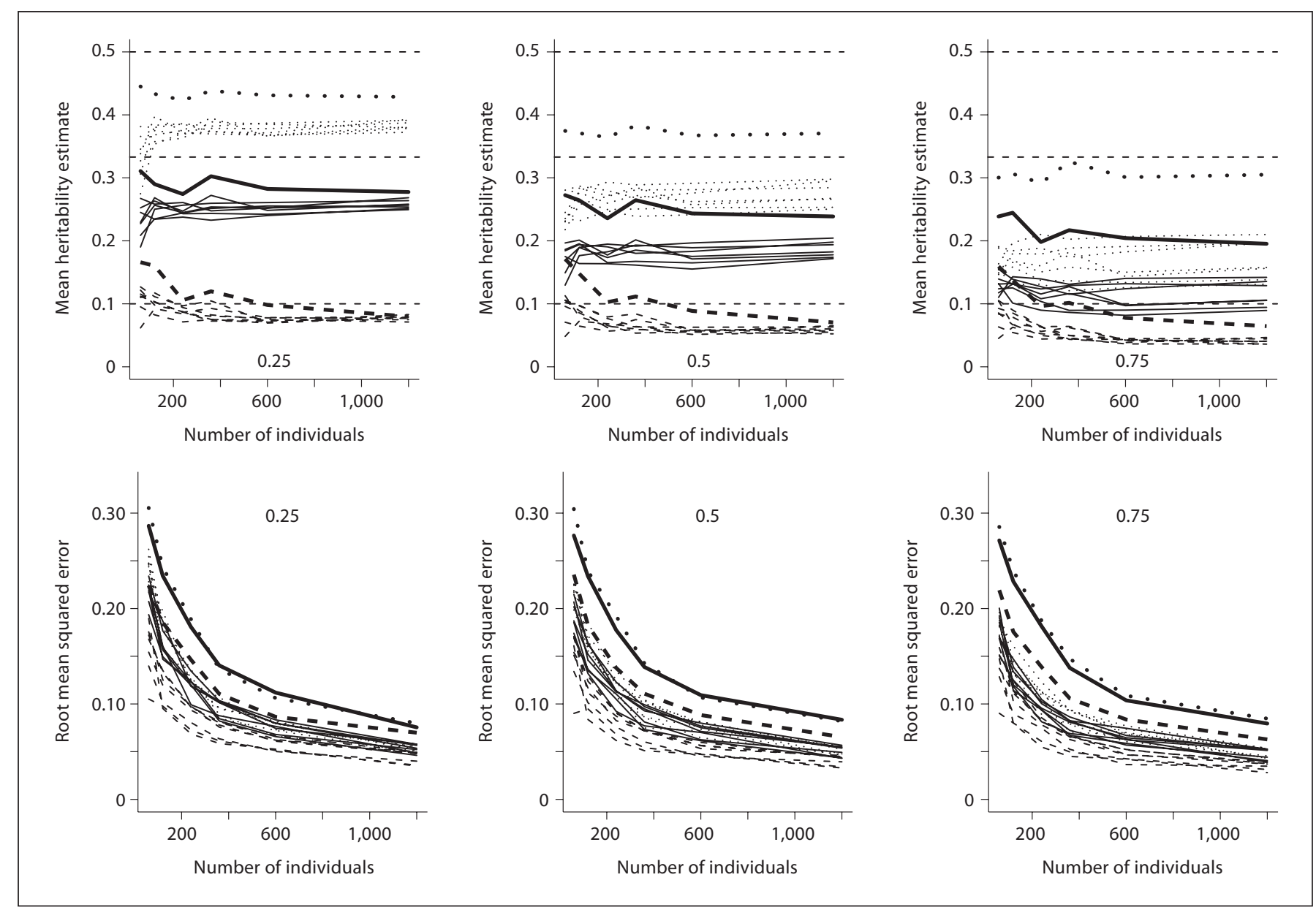

Fig. 6. Mean heritability estimates and root mean squared error of the narrow sense heritability estimates for eight different pedigree structures (a)-(h) (see fig. 1) and three levels of heritability $\left(h^{2}=0.1\right.$ (solid line), 0.33 (dashed line) and 0.5 (dotted line)). Simulated data include a dominance effect accounting for 25, 50 or $75 \%$ of the total genetic variation (columns 1-3 respectively) but the heritability estimates are estimated from an additive model that does not accommodate dominance effects. The bold lines correspond to the sib pair pedigree structure (a). All results are based on 1000 simulated datasets.

\section{Mixed Pedigrees Datasets}

In most real situations data consists of pedigrees of various structures. A dataset consisting of one of each of the 8 pedigree structures shown in figure 1 was simulated in order to compare the results of a mixed pedigree dataset with the datasets of a single pedigree structure. The combined dataset contains information on 61 individuals, which is comparable to the base sample size used in the simulations above. The results for the mixed pedigrees dataset generally resemble the results shown for the first cousins pedigrees $(\mathrm{g})$ in figures 4, 5 and 6 (data not shown). In other words, a few large pedigree structures appear to be sufficient to stabilize the variance components estimates.

The Impact of Pedigree Structure on Heritability

\section{Discussion}

In this paper I have investigated the impact of pedigree structure on precision and accuracy of heritability estimates. Eight different pedigree structures are examined both analytically and through simulations.

Most of the heritability information from human pedigree structures comes from parent offspring regression and the variation between and within full sib families. Falconer and Mackay [1996, equation 10.10] present explicit formulas for the heritability sampling variance for simple pedigree structures, and they show how the sampling variance from full sib families is twice as precise as the sampling variance from half sib families. Similar pre-

Hum Hered 2009;68:243-251 
dictions can be made for other types of (simple) pedigrees, see Visscher [2004] for a comparison of the sampling variance obtained from monozygotic and dizygotic twins in a variance component setup identical to the one described here. Hill and Nicholas [1974] show that the correlation between heritability estimates from mid-parent regression and estimates from full sib correlations are not trivial, and that pedigrees including information on both parents and offspring are more informative than pedigrees based on offspring alone. These results suggest that extended pedigrees may provide very little additional information about the heritability relative to the simpler nuclear family, and that is consistent with the results shown in figures 2,4 , and 6 .

Estimates based on fullsib pairs alone cannot properly determine the dominance effects as the dominance effects are confounded with the additive effects. Covariance matrices from fullsib pairs all have homogeneous off-diagonal entries so $\Phi_{i}$ and $\Delta_{7 i}$ (and also a shared environment covariance structure if that is included in the model) can not be separated.

Figures 2, 4, and 6 provide consistent results that show that - except for datasets consisting solely of sib pairs there may be virtually no impact of pedigree structure on the precision and accuracy of heritability estimates when the heritability is estimated using an additive model (1). Sib pair datasets have markedly higher heritability sample variance than the remaining pedigree structures (all 3 figures) but the sib pair data yield the same estimates as the other pedigree structures.

The heritability estimates are generally too small when the data contain a dominance effect but the model only accommodates an additive effect (fig. 6). This downward bias is caused by the model not being able to account for the dominance effects present in the dataset and the bias increases with increasing dominance effect. Sib pair pedigrees provide relatively larger heritability estimates that are closer to the true value. In this case however, the sib pair pedigrees fares better simply because the additive and dominance effects are virtually indistinguishable based on sib-pair covariances alone and therefore any dominance effects are easily classified as additive effects.

The results are somewhat different when data contain a dominance effect and the correct model is used to estimate the (broad sense) heritability (fig. 5). Here, there is substantial differences in the accuracy of heritability where some pedigree structures quickly converge to the correct heritability while others require notably larger sample sizes. The full sib pair pedigrees perform quite well with respect to accuracy and it is actually first and second cousin pedigrees (i.e., pedigrees $(\mathrm{g})$ and $(\mathrm{h})$ from fig. 1) that show the largest bias. This bias is caused by the lack of information about the dominance effect in those pedigrees. Although these pedigrees are fairly large there is only one relationship - the full sibling pair - that provides any information about the dominance effects. Thus, there is little information to distinguish dominance from additive effects and this results in an inflated estimate of the dominance effects. However, it should be emphasized that there is no difference in root mean squared error among the different pedigree structures except for first and second cousin pedigrees which have somewhat larger MSE than the other pedigrees. This is identical to the results seen in figures 2,4 , and 6 .

It is worth noting that even with very large sample sizes (e.g., 1200 individuals) there is still a large MSE even for the more complicated pedigree structures. The reason for the imprecision of heritability estimates is that the heritability is calculated from the estimated variance parameters and the variance of variances needs large number of observations before it stabilizes. Consequently, it is quite costly to obtain very precise estimates of the heritability, and the results from figure 4 suggests that even with 400 individuals the heritability estimate is on average off by 0.10 !

Pedigree size also influences the sample variance of the heritability, but a pedigree consisting of four full siblings show virtually the same MSE as the more complicated pedigree structures. Thus, pedigree size may be important for heritability precision but not the exact pedigree structure. The only place where pedigree structure appears to have a substantial impact is on the broad sense heritability (fig. 5) when data contains a dominance effect. Not surprisingly, the simulations suggest that the small-sample bias of broad sense heritability is reduced if pedigrees with several relationships that provide information about the dominance correlation are sampled.

Although there may generally be little impact of pedigree structure on the heritability estimates based on an additive model as shown in figures 2,4 , and 6 , the pedigree structure can have a huge impact on quantitative trait locus (QTL) detection (Williams and Blangero, 1999). Variance component QTL linkage analysis use the information from the locus-specific correlations among individuals to identify any excess correlation due to the QTL. A sib pair pedigree has only one relationship (that of the two siblings) that can provide information about the QTL, while a second cousin pedigree has numerous 
pairwise comparisons that provide information about the excess correlations potentially caused by the QTL.

De Andrade and Amos [2000] discuss ascertainment issues in variance component models. They consider two ascertainment schemes: conditioning on the trait value of the proband and conditioning on the probability that the trait value in a proband is above a pre-specified threshold. De Andrade and Amos [2000] conclude that failure to correct for ascertainment affects the estimates of the variance component such that heritability estimates are too low in the presence of a common major allele. The downward bias of the heritability estimates was removed when ascertainment correction was used in the analyzes. Clearly, when estimating variance components and heritability the correct model should always be employed and that includes a proper ascertainment correction whenever that is known.

In conclusion, the present study suggests that pedigree structure is not so important for heritability studies as long as the sampled pedigrees are of moderate size. Also, it is unnecessary to sample complicated pedigrees instead of, say, nuclear families, since there is no practical differ- ence in the MSE between large, complex and pedigrees of moderate size. However, if only sib pairs are available it is still feasible to estimate the heritability since the bias for sib pair pedigrees is the same as for the more complex pedigrees except when both the sample size and heritability are low. The additive model heritability estimates are downward biased in the presence of dominance effects. However, the heritability estimates can still be used in the planning of linkage or association studies since the estimated heritability will be too low, which in turn results in a demand for an increased number of pedigrees necessary to obtain a given power.

The simulation results also suggest that a large number of individuals are required to obtain a good precision of the heritability and that the improvement inMSE of the heritability estimate does not outweigh the cost of sampling additional pedigrees (e.g., 200-400 individuals are generally enough to get a reasonable stable estimate).

Simulation and estimation was done using the PediPet source code, which can be downloaded from www.statistics.life.ku.dk/ ekstrom/pedipet/.

\section{References}

Almasy L, Blangero J: Multipoint quantitativetrait linkage analysis in general pedigrees. Am J Hum Genet 1998;62:1198-1211.

$\checkmark$ de Andrade M, Amos CI: Ascertainment issues in cariance components models. Genet Epidemiol 2000;19:333-344.

Falconer DS, Mackay TFC: Introduction to Quantitative Genetics, ed 4. Longman, Harlow, England, 1996.

-Hill WG, Nicholas FW: Estimation of heritability by both regression of offspring on parent and intra-class correlation of sibs in one experiment. Biometrics 1974;30:447-468.
Hopper JL, Mathews JD: Extensions to multivariate normal models for pedigree analysis. Ann Hum Genet 1982;46:373-383.

-Hsu FC, Zaccaro DJ, Lange LA, Arnett DK, Langefeld CD, Wagenknecht LE, Herrington DM, Beck SR, Freedman BI, Bowden DW, Rich SS: The impact of pedigree structure on heritability estimates for pulse pressure in three studies. Hum Hered 2005;60:63-72.

Jacquard A: The Genetic Structure of Populations. Springer-Verlag, N.Y., 1974.

-Lange K, Westlake J, Spence MA: Extensions to pedigree analysis III. Variance components by the scoring method. Ann Hum Genet 1976;39:485-491.
Lynch M, Walsh B: Genetics and Analysis of Quantitative Traits. Sinauer Associates, 1998.

Mallinckrodt CH, Golden BL, Reverter A: Approximate confidence intervals for heritability from method $\mathrm{R}$ estimates. J Anim Sci 1997;75:2041-2046.

Visscher P: Power of the classical twin design revisited. Twin Res 2004;7:505-512.

Williams JT, Blangero J: Power of variance component linkage analysis to detect quantitative trait loci. Ann Hum Genet 1999;63:545563. 